\title{
Evaluation of Tortuosity in Cemented Sand Using X-Ray Computed Microtomography
}

\author{
Junil Pae ${ }^{1}$ and Juhyuk Moon ${ }^{2}$ \\ ${ }^{1}$ Department of Civil and Environmental Engineering, Seoul National University, 1 Gwanak-ro, \\ Gwanak-gu, Seoul, 08826, Republic of Korea, sdc03055@snu.ac.kr \\ 2 Department of Civil and Environmental Engineering, Seoul National University, 1 Gwanak-ro, \\ Gwanak-gu, Seoul, 08826, Republic of Korea, juhyukmoon@snu.ac.kr
}

\begin{abstract}
Cemented sand is a common way for soil stabilization or ground improvement. Tortuosity is an important parameter that has a significant impact on flow and transport characteristics of porous media and related to permeability and diffusion coefficient. This study aims to quantitatively investigate the tortuosity of the cemented sand with different cement content using X-ray computed tomography (CT) based random walk simulation. The cemented samples were scanned using micro$C T$ and converted to $3 D$ pore space through image processing. Random walk simulation was applied to the reconstructed $3 D$ pore space to calculate the tortuosity. Higher cement content gave an increase in tortuosity and a decrease in porosity. This indicates that the addition of cement makes pore space more tortuous and decreases both permeability and diffusion coefficient which are being treated as important parameters for durability of construction materials.
\end{abstract}

Keywords: Cemented Sand, Tortuosity, X-Ray Microtomography, Random Walk Simulation.

\section{Introduction}

Soil stabilization by cement mixing is one of the common methods to improve natural soil. Generally, cemented sand which is produced by mixing cement, sand, and water, has greater strength than natural soil. Ordinary Portland Cement is widely used in soil stabilization and many researches were carried out to investigate its mechanical (Consoli, Viana da Fonseca, Cruz, and Heineck, 2009; Wang and Leung, 2008). Pore network in cementitious material is an important factor that affects many mechanical properties including strength and stiffness as well as durability. However, it is difficult to quantitatively analyse due to its complexity thus several experimental parameters like porosity, permeability, and diffusivity are frequently being used to understand pore network and resulting durability, indirectly. Therefore, the knowledge of tortuosity for cemented sand is strongly required for designing not only the cement content for soil stabilization but also evaluating life span of cementitious materials under various environments.

X-ray Computed Tomography (CT) has been utilized to examine the microstructure of porous media. X-ray $\mathrm{CT}$ is a non-destructive technique that allows X-rays to pass through the material at different angles and measures the attenuation using the director. The key advantage of X-ray CT is that it is a unique way to obtain three-dimensional microstructure at specific resolution. This study aims to investigate the tortuosity of the cemented sand with different cement content from the X-ray CT based random walk simulation. 


\section{Background of Definition of Tortuosity}

Tortuosity is a meaningful parameter that has a significant impact on flow and transport characteristics of porous media. The classic definition of tortuosity $(\tau)$ is given as the ratio of the average lengths of the actual paths that fluid or electric particles migrate from one point to the final point through the pore space of a porous medium to the corresponding straight and shortest distance between these points. In this regard, tortuosity is always greater than or equal to 1 . The importance of tortuosity can be observed in various engineering applications (e.g., diffusion, mass transfer and separation of mixtures) (Boudreau, 1996; Champoux and Allard, 1991; Thorat et al., 2009).

In this study, tortuosity is treated as a property of the porous media which can be calculated from 3D micro-geometry of the pore structure. For instance, the tortuosity in cementitious materials has been evaluated by random walk simulation in the 3D pore structure obtained by X-ray microtomography (Promentilla, Sugiyama, Hitomi, and Takeda, 2009;

Provis, Myers, White, Rose, and Van Deventer, 2012).

Diffusion tortuosity is defined as a ratio of the self-diffusion coefficient $\left(D_{0}\right)$ of nonsorbing walkers in free space to the long-time self-diffusion coefficient $\left(D_{\infty}\right)$ of these walkers in pore space. The self-diffusion coefficient is estimated from mean square displacement (MSD) from the movement trajectories these random walkers as a function of time. The timedependent diffusion coefficient $\mathrm{D}_{\mathrm{t}}$ associated with the random Brownian motion of molecules in porous media. If diffusion time is great enough, it will be a non-zero value that can represent the entire pore characteristic because
approaches zero in a closed pore space. Th
molecule only in a donnected pore structure
defined as the diffusion tortuosity.
The formation factor $(F)$ can be calculated f
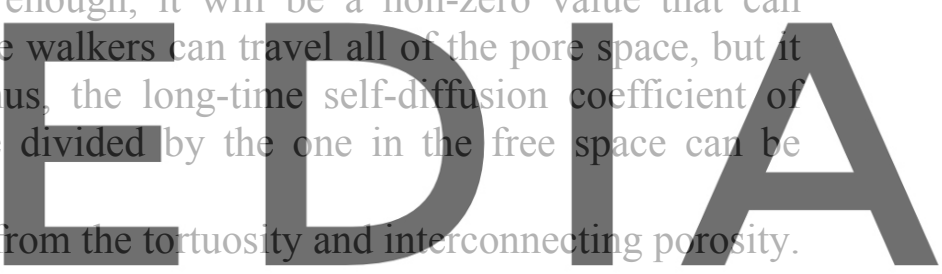

\section{$F=\frac{\tau_{D}}{\varnothing}$}

(1)

Register for free at http.s//www scipedia.com to download the version without the watermark By Nernst-Einstein relation, the relative diffusibility, formation factor, porosity and tortuosity are related to each other.

$$
\frac{D_{e}}{D_{f}}=\frac{1}{F}=\frac{\emptyset}{\tau_{D}}
$$

where $D_{e}$ is the intrinsic diffusion coefficient by using Fick's law, and $D_{f}$ is the diffusion coefficient in the free fluid (Promentilla et al., 2009).

\section{Materials and Methods}

\subsection{Sample Preparations and Image Acquisition}

The specimens of cemented sand used in this study were prepared by mixing Jumunjin standard sand and ordinary Portland cement Type 1 . The sand/cement ratio of each specimen is $0 \%, 1 \%, 3 \%, 5 \%, 7 \%$ and water content which means the ratio of mass of water to solids (includes both sand and cement) is 0.1 . The mix design is shown in Table 1. Samples are prepared in PVC molds and cured at $20^{\circ} \mathrm{C}$ for 7 days. One specimen per each mix was 
analyzed by the CT and one volume of interest (VOI) per specimen was selected for image processing as described below.

Table 1. Mix design of cemented sand.

\begin{tabular}{cccc}
\hline $\begin{array}{c}\text { Sand } \\
(\mathrm{g})\end{array}$ & $\begin{array}{c}\text { Cement } \\
(\mathrm{g})\end{array}$ & $\begin{array}{c}\text { Water } \\
(\mathrm{g})\end{array}$ & $\begin{array}{c}\mathrm{w} / \mathrm{c} \\
\text { ratio }\end{array}$ \\
\hline 1000 & 0 & 100 & - \\
\hline 1000 & 10 & 101 & 10.1 \\
\hline 1000 & 30 & 103 & 3.43 \\
\hline 1000 & 50 & 105 & 2.10 \\
\hline 1000 & 70 & 107 & 1.53 \\
\hline
\end{tabular}

The X-ray imaging of specimens was performed by the Skyscan micro-CT (Skyscan1272, Bruker, Belgium). Acquired reconstructed image from tomographic data has $4904 \times 4904$ pixels and pixel size is $5 \mu \mathrm{m}$ by $5 \mu \mathrm{m} .3280$ layers of X-ray projection image are obtained with exposure time of $5000 \mathrm{~ms}$ and an angle step of $0.1^{\circ}$.

\subsection{Construct of 3D Pore Space}

The VOI which is the portion of the full data set chosen for segmentation is necessary due to some limitations of memory during computation. The size of VOI should also be large enough to eliminate the introduction of errors resulting fron the cropping images (i.e., the finite size). It has been least $100 \mu \mathrm{m}^{3}$, or 3 to 5 times larger than the for hardened OPC pastes, to eliminate finite size al., 2012). In this study, $1000 \times 1000$ pixels o:

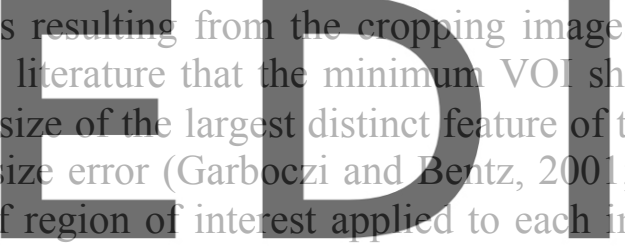
s (i.e., the
ould be at
the sample
; Provis et
mages and then it merged to $1000^{3}$ voxels of VOI which is equal to 3D pore space which satisfies the

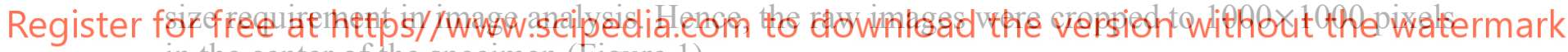
in the center of the specimen (Figure 1).

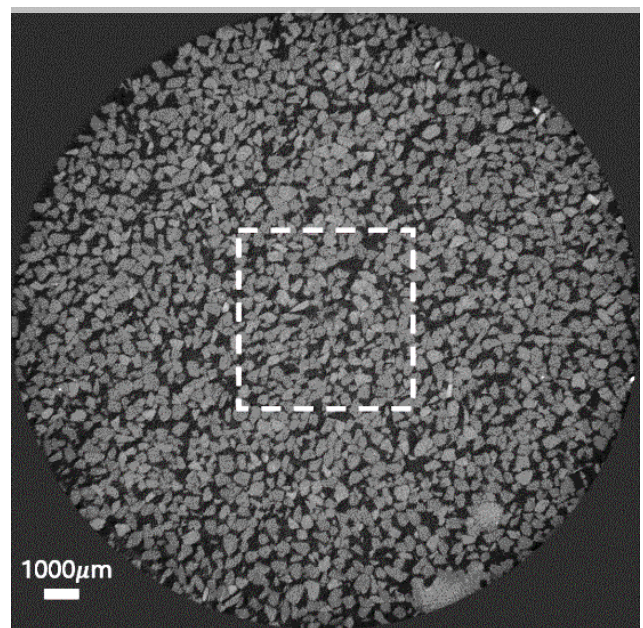

Figure 1. A slice through the full tomographic reconstruction of the 7-day $1 \%$ cemented sand specimen. White dotted area shows the size of the VOI selected. 
First, Gaussian blur and anisotropic diffusion were adjusted in sequence in order to smooth the raw images while remaining the narrow pore space between sand particles. This procedure enhances the accuracy of watershed algorithm applied later. Then, segmentation is carried out to separate pore and solid due to the difficulty of distinguish sand phase and cement phase in $\mathrm{X}$-ray image at a resolution lower than $1 \mu \mathrm{m} /$ pixel. Thresholding value in grayscale is selected as 5 greater than the value calculated by Otsu method in entire dataset due to eliminate the noises in pores space (Otsu, 1979). In addition, the narrow pores between sand particles containing lots of noises could be converted to pore after segmentation. Sand and pore phase are modified to white and black, respectively.

After pore segmentation, 1000 slices of $2 \mathrm{D}$ images are merged to $3 \mathrm{D}$ pore space to

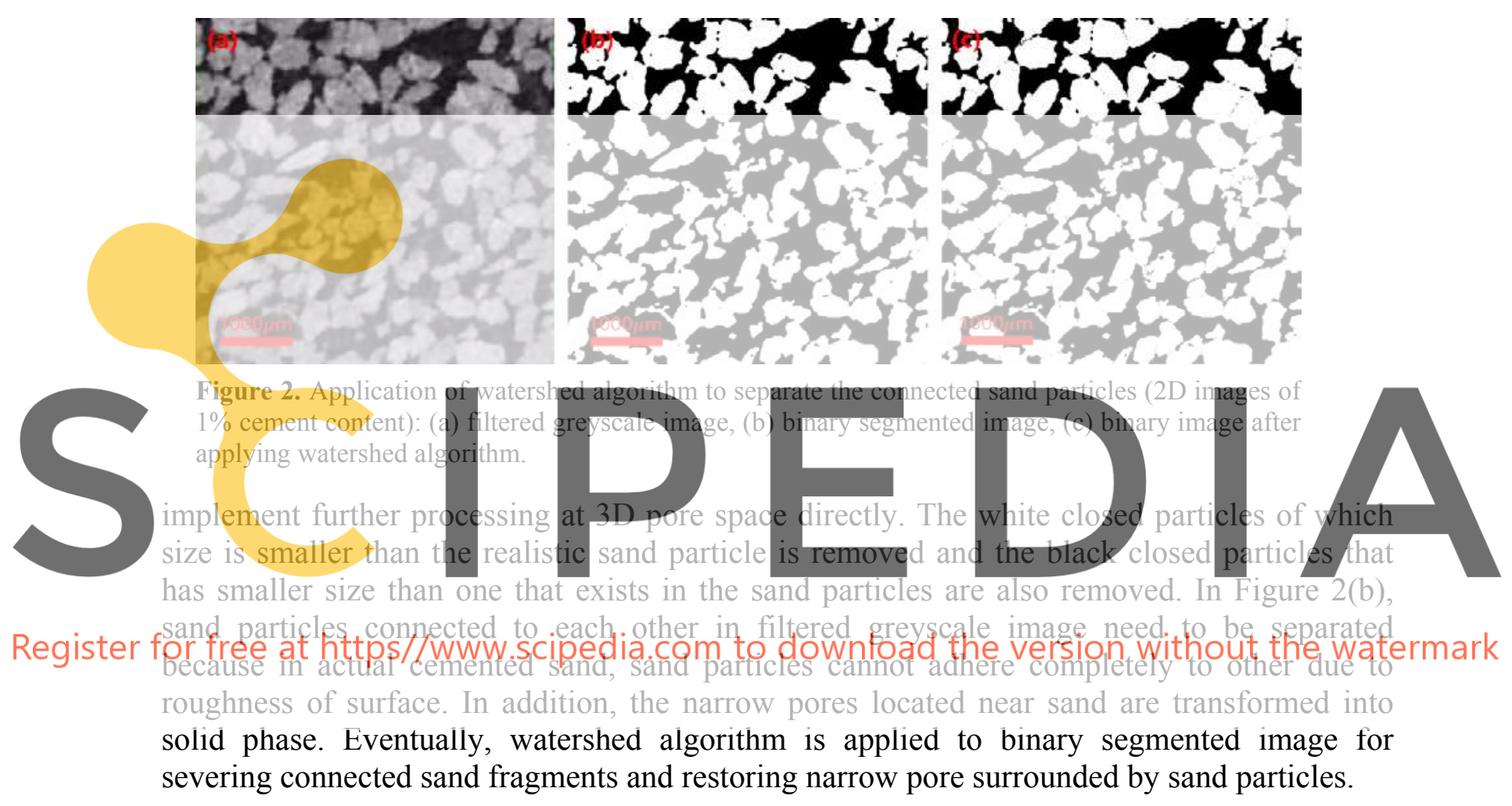

\subsection{Particle Size Distribution of Sand}

Particle size distribution (PSD) of sand is considered to verify the used image processing method. Sieve analysis was performed for the identical sand to gain PSD data following ASTM D422. In 3D pore space after applying watershed algorithm, all of the sand particles exist independently. It allows to calculate the volume of each separated sand particles by using cluster labeling. PSD is also obtained from these volume data by calculating equivalent diameter. Therefore, the accuracy of segmented 3D pore structure can be evaluated by the comparison of the PSDs obtained from the sieve analysis and image processing.

\subsection{Tortuosity Calculation From Random Walk Simulation in 3D Pore Space}

A 3D random walker simulation (Nakashima and Kamiya, 2007) implanted in MATLAB was used to compute the MSD of random walkers in the segmented pore space. Mirror image 
boundary conditions were employed to the original pore space, resulting to an infinitely expanded pore structure where simulations are performed with preserving the continuity of inter-connected pore network. The average displacement of the walkers was plotted as a function of the number of the timesteps and the tortuosity was calculated from the inverse of the slope of this curve.

To execute the random walker simulation, a random pore voxel in the inter-connected pore network is chosen as the initial position of each walker. In each timesteps, the one was selected among the six voxels which are face-to-face connected with the random walker. Then the random walker migrates to the selected voxel when it has been identified as pore space. The diffusion tortuosity is defined as the ratio of the self-diffusion of a walker in free space to the self-diffusion of a walker in the pore space such that:

$$
\tau_{D}=\frac{D_{0}}{D_{\infty}}=\left[\lim _{t \rightarrow \infty} \frac{\mathrm{d}\left\langle r^{2}(t)\right\rangle}{\mathrm{d} t}\right]^{-1}
$$

where $D_{0}$ is the self-diffusion coefficient of a walker in free space, $D_{\infty}$ is the self-diffusion coefficient of a walker in the pore space, and $\left\langle r^{2}(t)\right\rangle$ is the average of MSD as a function of a time. By using Eq. (3), restricted diffusion that reflects the geometry of the entire pore space can be defined as the diffusion tortuosity. On such time scales, the MSD starts to increase linearly with the number of timesteps due to an ample time for random walkers to travel the entire pore space, leading to constant calculated tortuosity.

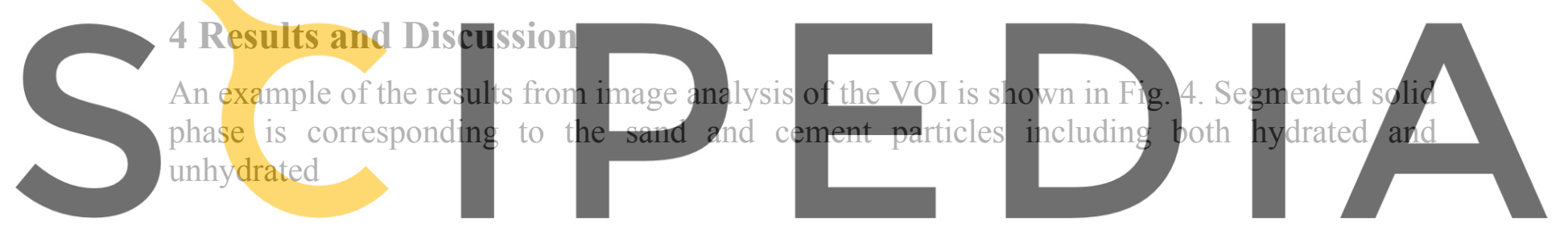

Register for free at htfps//WwW.scipedia.com to downioad the version without the watermark
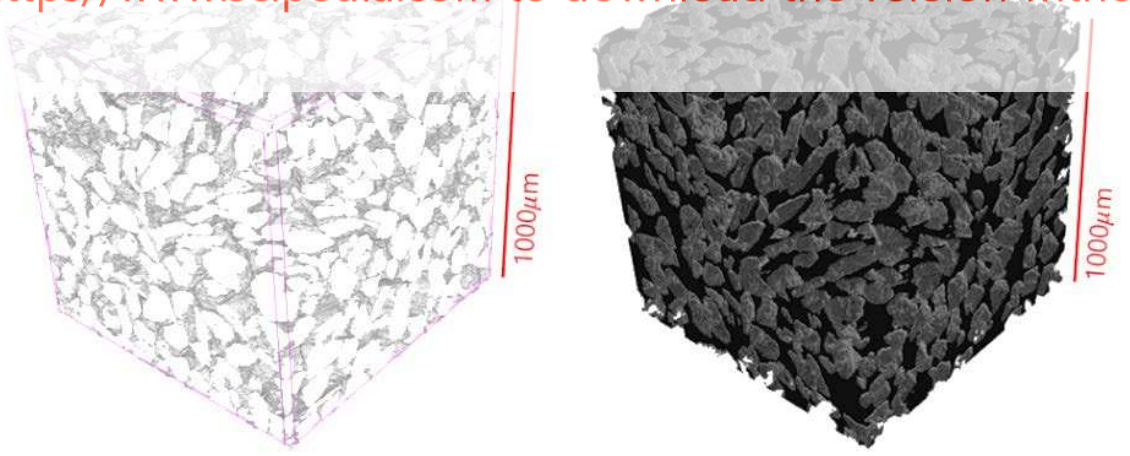

Figure 3. Visualization of pore space from microtomographic images of the $1 \%$ cemented sand sample $\left(\mathbf{1 0 0 0}^{3}\right.$ voxels). (a) binary image of segmented solid phase. (b) binary image of segmented pore phase.

\subsection{Porosity}


The data presented in Figure 4 shows that the segmented porosity decreases as the cement content increases. This trend holds for all samples with one exception of $0 \%$ cement sample. It showed a lower porosity than $1 \%$ cement sample. The $0 \%$ cement sample has only two phases which are sand and pore besides other samples have three phase including cement hydrate. This different number of phases can introduce the difference in overall shape of the greyscale histograms that affects to the threshold value selection in segmentation process and results underestimated porosity in the $0 \%$ cement sample. As cement content increases, porosity decreases because cement particles fill more pore space which are formed by packing of sand particles. The inter-connected porosity is completely equal to general porosity. This implies that all pores found in cemented sample are connected.

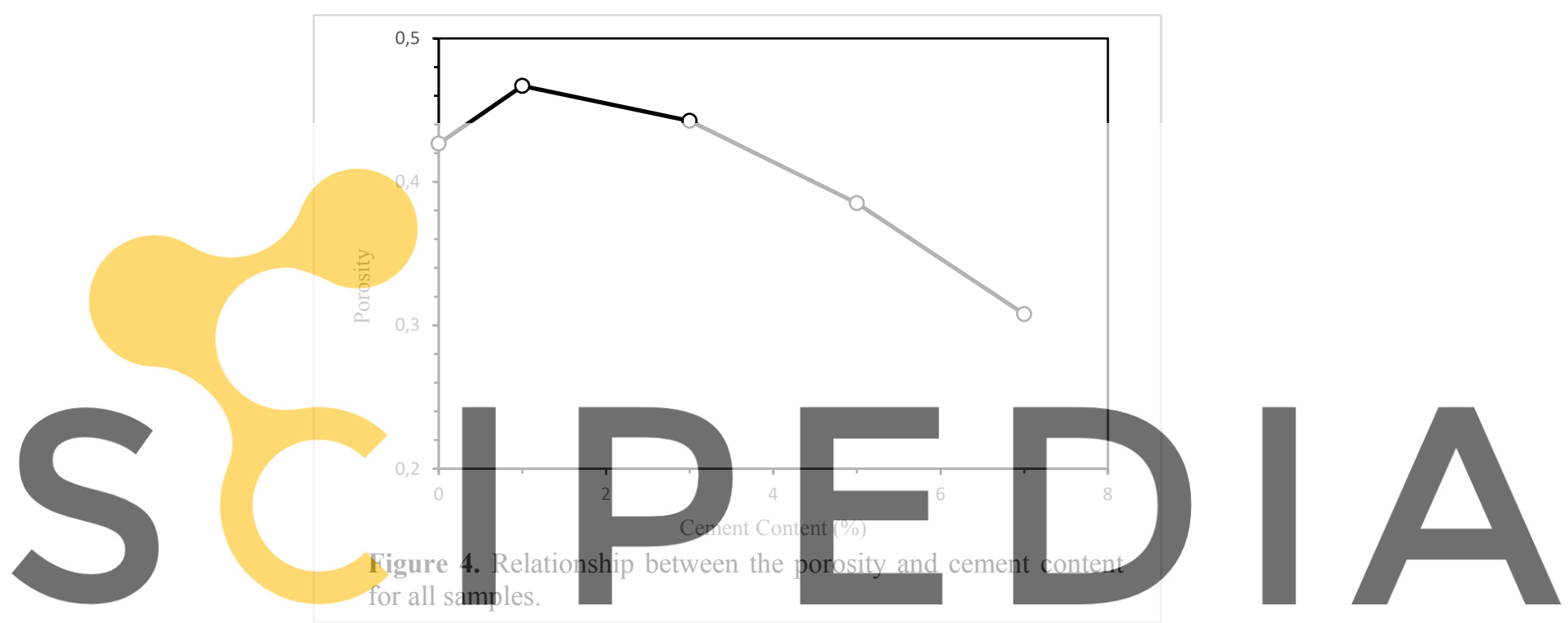

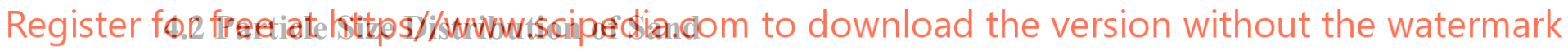

Figure 5 compares the PSD of raw sand from sieve analysis and those of segmented solid obtained by image analysis. The $0 \%$ cement sample and sieve analysis are subject to investigate the accuracy of the microtomographic data in compare with actual data (i.e., sieve result). There is a consistent horizontal shift between PSDs. The constant horizontal shift can be introduced due to the discrepancy of the diameter used to plot. For instance, volumeequivalent diameter was applied to generate PSDs for all cemented sand samples, whereas the diameter used for sieve analysis was a maximum diameter that a sand particle had to pass through the specific sieve.

For the $1 \%, 3 \%, 5 \%$, and $7 \%$ cemented sand samples, the size of segmented solid particles increased as the cement content increased. This is expected because cement hydrate adheres to sand particles. The size of segmented particle size is the largest for $7 \%$ cemented sand sample because of the largest amount of cement used. 


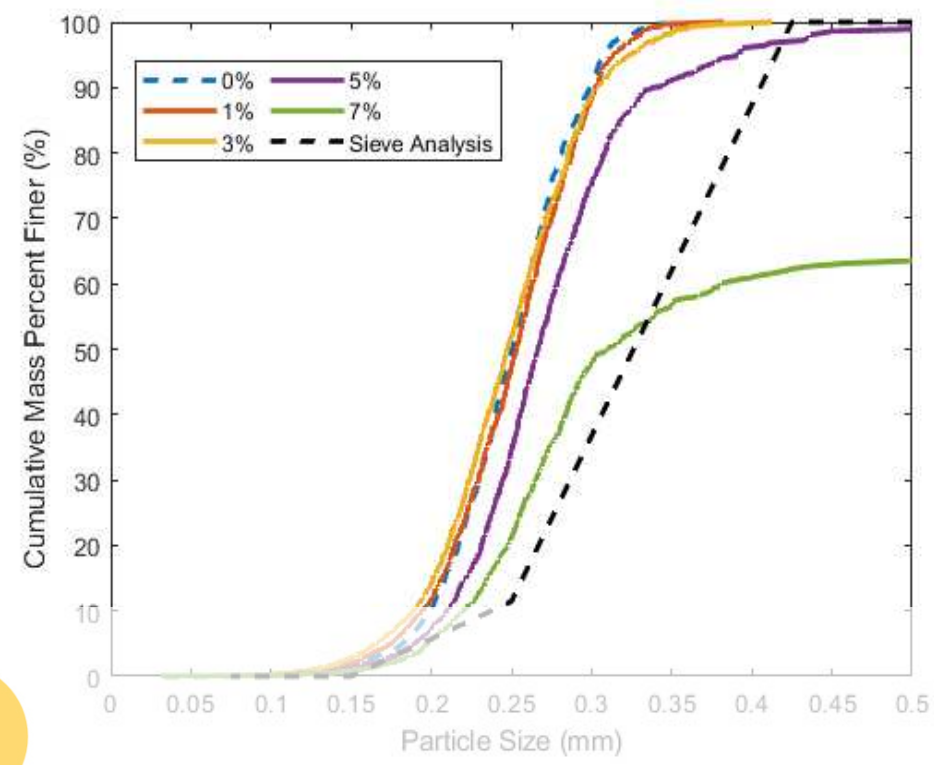

Figure 5. Particle size distribution of sand particles for all cemented sand sample and the data obtained by sieve analysis.

\subsection{Tortuosity}

Figure 6 shows the diffusion

simulation on the segm

increased with cement

tortuous as cement added in the system. Cement hydration
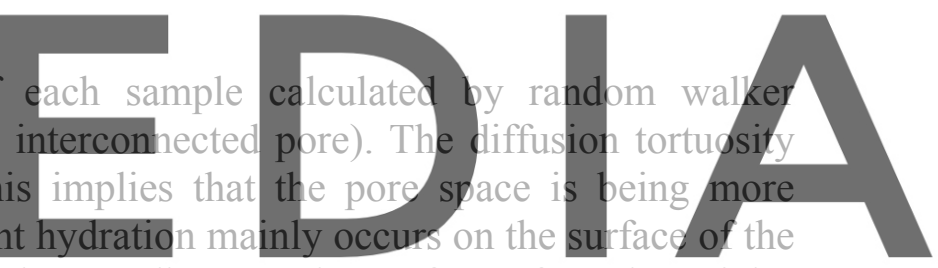

existing sand structure. Therefore, cement hydrates adhere to the surface of sand particles

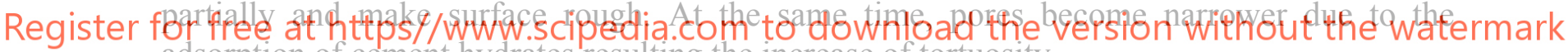
adsorption of cement hydrates resulting the increase of tortuosity.

Figure 7 presents the formation factor as a function of cement content. The formation factor increased as cement content increased except for $0 \%$ cement sample which has intrinsically different solid system (i.e., two phases vs. three phases) as previously discussed. Nevertheless, obtained trends of increasing tortuosity and formation factor clearly suggest that permeability and diffusion coefficient should decrease with cement addition. Thus, it can be quantitatively inferred that the addition of cement in cemented sand is an effective way for controlling permeability and diffusion.

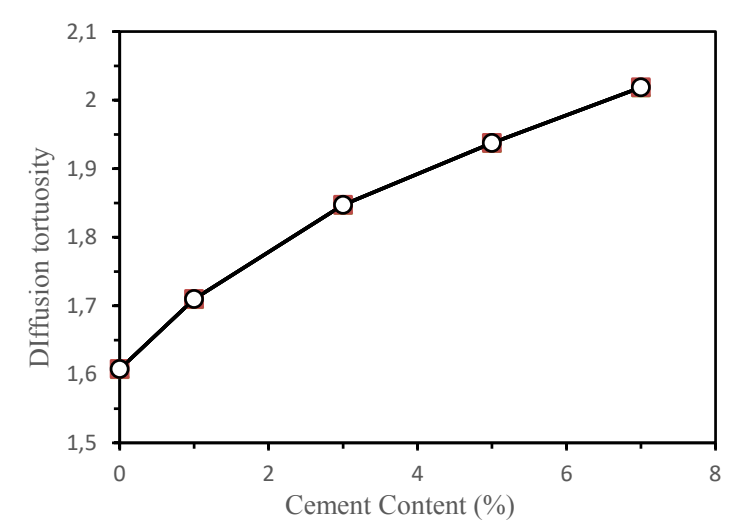

Figure 6. Relationship between the diffusion tortuosity and cement content for cemented sand sample.

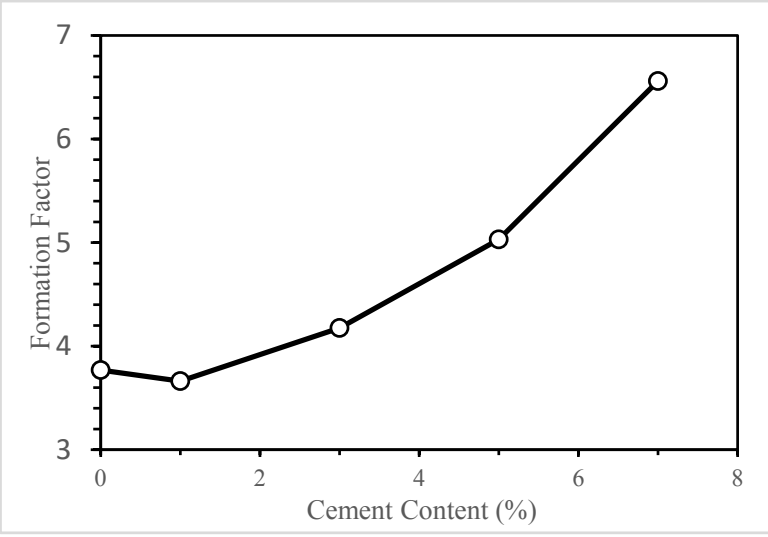

Figure 7. Relationship between the formation factor and cement content for cemented sand sample. 


\section{Conclusion}

The porosity and tortuosity parameters obtained from the tomography data here provide an indication of the pore network geometry and microstructure of cemented sand. Higher cement content gave an increase in tortuosity and a decrease in porosity. This indicates that the small addition of cement hydrate in compacted sand, makes pore space more tortuous.

This study has provided the methodical three-dimensional analysis of cemented sand by Xray microtomography. There remain difficulties in the accurate identification of pore and solid regions in cemented sand due to the low X-ray absorption. Phase contrast tomography proposes the potential in this area, but there is integral limitation due to microscale VOI which is small to represent intrinsic parameters of cemented sand. Nevertheless, the data presented in this study provide invaluable information regarding pore network, porosity and tortuosity, and suggest the novel method for future developments which can be led to further understanding on durability of solid materials.

\section{Acknowledgement \\ This work was supported by the BK4 funded by the Ministry of Education, Republic of Korea. \\ ORCID}

Junil Pae: http://orcid.org/0000-0002-1494-5270

Juhyuk Moon: http://orcid.drg/0000-0003-44>3-4308

References

Boudreau, B. P. (1996). The diffucive tortuosity

Cosmochimica Acta, 60(16), 3139-3142.
Champoux, Y. and Allard, J. F. (199:). Dynamic torthosity and bulk modulus in air-saturated porous media. Journal of applied physics, 70(4), 1975-1979.

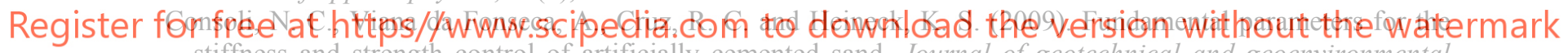
stiffness and strength control of artificially cemented sand. Journal of geotechnical and geoenvironmental engineering, 135(9), 1347-1353.

Garboczi, E. J. and Bentz, D. P. (2001). The effect of statistical fluctuation, finite size error, and digital resolution on the phase percolation and transport properties of the NIST cement hydration model. Cement and Concrete Research, 31(10), 1501-1514.

Nakashima, Y. and Kamiya, S. (2007). Mathematica programs for the analysis of three-dimensional pore connectivity and anisotropic tortuosity of porous rocks using X-ray computed tomography image data. Journal of Nuclear Science and Technology, 44(9), 1233-1247.

Otsu, N. (1979). A threshold selection method from gray-level histograms. IEEE transactions on systems, man, and cybernetics, 9(1), 62-66.

Promentilla, M. A. B., Sugiyama, T., Hitomi, T. and Takeda, N. (2009). Quantification of tortuosity in hardened cement pastes using synchrotron-based X-ray computed microtomography. Cement and Concrete Research, 39(6), 548-557.

Provis, J. L., Myers, R. J., White, C. E., Rose, V. and Van Deventer, J. S. (2012). X-ray microtomography shows pore structure and tortuosity in alkali-activated binders. Cement and Concrete Research, 42(6), 855-864.

Thorat, I. V., Stephenson, D. E., Zacharias, N. A., Zaghib, K., Harb, J. N. and Wheeler, D. R. (2009). Quantifying tortuosity in porous Li-ion battery materials. Journal of Power Sources, 188(2), 592-600.

Wang, Y. and Leung, S. (2008). Characterization of cemented sand by experimental and numerical investigations. Journal of geotechnical and geoenvironmental engineering, 134(7), 992-1004. 